\title{
ELECTRONIC STRUCTURE OF DILUTED SEMIMAGNETIC SEMICONDUCTOR (Cd,Co)Se
}

\author{
V. CHÁB, J. MAŠEK \\ Institute of Physics, Czecho-Slovak Academy of Sciences \\ Na Slovance 2, 18040 Prague 8, Czecho-Slovakia \\ B.A. ORLOWSKI AND B.J. KowalsKI \\ Institute of Physics, Polish Academy of Sciences \\ Al. Lotników 32/46, 02-668 Warszawa, Poland
}

\begin{abstract}
The electronic structure of a new semimagnetic semiconductor (Cd,Co)Se is studied by UPS and calculated by a tight-binding version of the disordered-local-moment theory. The theory accounts for the chemical disorder and for electron interactions within the Co $3 d^{7}$ shells. Both theory and experiment show Co $3 d$ states deep in the valence band and also at the band edge. The last states seem to be responsible for unique properties of the Co-based semimagnetic semiconductors.
\end{abstract}

PACS numbers: 75.10.Lp, 75.50.Pp, 79.60.Eq

\section{Introduction}

The traditional semimagnetic semiconductors (SMSC) are obtained by substituting manganese in the cation sublattice of semiconducting II-VI compounds. An extensive experimental and theoretical study of these materials showed that their specific magnetical, optical and electrical properties are largely determined by the presence of the Mn $3 d$ electrons and their strong interactions. The crucial point, i.e. the position of the $d$ states in the valence band and the degree of their hybridization with the band states was resolved by using the photoemission experiments supported by theoretical calculations (e.g. Ref. [1]).

In this paper, we turn to a new family of the semimagnetic semiconductors containing cobalt instead of manganese. They have been recently subjected to detailed magnetical investigations which show that the Co-based SMSC have much larger exchange constants than their Mn-based conuterparts [2]. Much less 
is known about the spectral properties of the SMSC with Co which are, however, very useful for understanding the electronic structure and the origin of enhanced magnetic interactions [3].

We take $\mathrm{Cd}_{1-x} \mathrm{Co}_{x} \mathrm{Se}$ as a representative example of the SMSC with cobalt and use the photoelectron spectroscopy [4] to determine the distribution of the Co $3 d$ states in the valence band. We profit from our previous experience with the study of the Mn-based systems and combine the photoemission experiment with the theoretical calculations of the valence density of states in $\mathrm{Cd}_{1-x} \mathrm{Co}_{x} \mathrm{Se}$ which makes us also possible to see the differences between the Co- and Mn-based SMSC.

\section{Theory}

The photoemission spectrum from a semimagnetic mixed crystal is given by a configurationally averaged density of states (DOS) in the valence band. We calculate it by using a tight binding version of the disordered-local-moment (DLM) theory [5] adapted to the case of diluted alloys [1,3].

The interacting electrons are described by a multisite degenerate Anderson Hamiltonian

$$
H=H_{\text {band }}+H_{\text {int }}+H_{\text {hybr }} .
$$

Its three parts correspond to electronic motion in the bands of the host CdSe crystal, electron-electron interactions within the $d$-shells of the Co ions, and the hybridization of the $d$ states with the bands. Within a static version of the DLM theory we apply the unrestricted Hartree-Fock approximation to the interaction part of the Hamiltonian, independently for each site occupied by a transition metal ion. We assume that, apart from the polarity of the exchange part, all local Hatree-Fock potentials are the same. The exchange potential, leading to a preferential occupation of the Co $d$ states by spin-up or spin-down electrons, changes randomly from site to site and results in a formation of randomly oriented local moments (paramagnetic state). With two possible polarities of the local exchange potentials, up and down, we describe the magnetically disordered phase of $\mathrm{Cd}_{1-x} \mathrm{Co}_{x} \mathrm{Se}$ as an effective ternary alloy $\mathrm{Cd}_{1-x} \mathrm{Co}_{x / 2}^{\dagger} \mathrm{Co}_{x / 2}^{\downarrow} \mathrm{Se}$.

The electronic structure of the alloy is calculated, as usual, by using the coherent potential approximation. We use its tight-binding version and assume, for simplicity, a mixed crystal with a zinc-blende structure. The details of the parametrization are given in another paper of this issue [6].

The calculated DOS for $\mathrm{Cd}_{0.9} \mathrm{Co}_{0.1} \mathrm{Se}$ is given in Fig. 1 (curve (b)). For reference, we show the density of states for a pure CdSe crystal (curve (a)), and also the results for $\mathrm{Cd}_{0.9} \mathrm{Mn}_{0.1} \mathrm{Se}$ (curve (c)). The gross features of the valence spectrum of both semimagnetic compounds are similar to the DOS of CdSe. The spectrum of $\mathrm{Cd}_{0.9} \mathrm{Co}_{0.1} \mathrm{Se}$ has two additional peaks deep in the valence band (binding energies $2.9 \mathrm{eV}$ and $3.5 \mathrm{eV}$ ) and another peak at the valence band top. The former peaks, similar to those in the Mn-based alloy, accumulate five majority spin electrons per Co atom. The last structure, unique to the Co-based semimagnetic semiconductors (cf. Ref. [3]), is formed by two remaining Co $3 d$ electrons with opposite spin. 


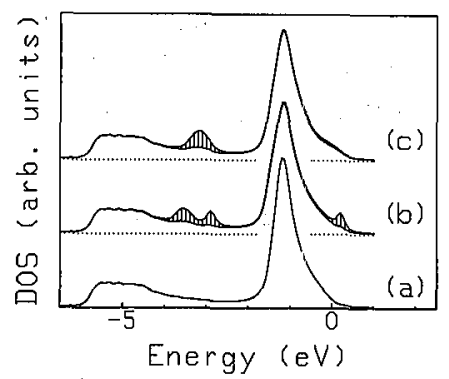

Fig. 1. Density of valence states of the zinc-blende CdSe crystal (a) and paramagnetic mixed crystals $\mathrm{Cd}_{0.9} \mathrm{Co}_{0.1} \mathrm{Se}$ (b) and $\mathrm{Cd}_{0.9} \mathrm{Mn}_{0.1} \mathrm{Se}$ (c). The contributions of the $d$ states are shaded. Notice that both mixed crystals remain semiconductors with Fermi energy at ca. $1.4 \mathrm{eV}$.

These states, close in energy to the anion $p$ states, hybridize efficiently with the bands; this results in an enhanced spin polarization of the band electrons and, consequently, to remarkably large exchange constant in $\mathrm{Cd}_{1-x} \mathrm{Co}_{x} \mathrm{Se}[2,6]$.

\section{Experimental results}

In order to find a contribution of $3 d^{7}$ electrons to the valence band of $\mathrm{Cd}_{1-x} \mathrm{Co}_{x} \mathrm{Se}$ alloys, He I photoelectron spectra were measured. The experiments were performed in an angle-resolved photoelectron spectrometer (VG ADES 400) at the basic pressure $10^{-10}$ Torr and with the energy resolution $\sim 0.1 \mathrm{eV}$. The samples $(x=0.0, x=0.12)$ were mechanically polished and the clean surface was prepared by Ar ion bombardment so that the measured spectra represent the optical density of states.

The experimental results are summarized in Fig. 1 of Ref. [4]. The valence band DOS of CdSe is dominated by two peaks at binding energies $-1.6 \mathrm{eV}$ and $-4.6 \mathrm{eV}$. The Co contribution to the valence band of CdSe does not change the spectrum at the first glance. The main peaks are slightly broadened in $\mathrm{Cd}_{0.88} \mathrm{Co}_{0.12} \mathrm{Se}$ and no new emission structures are visible in the rough data. Only the difference spectrum shows the $3 d^{7}$ electron contribution to the DOS. Two broad maxima were found - one at the edge of the valence band $(-0.8 \mathrm{eV})$ and the second nearly in the minimum of the CdSe density of states $(-3.5 \mathrm{eV})$. Thus, the Co $3 d$ electrons contribute to the valence band of mixed crystal in a similar way as the Fe $3 d$ electrons in Fe-based SMSC [4,7], in contrast to the case of the traditional Mn-based systems $[4,7]$.

\section{Summary}

Both theory and photoemission spectra show that there is an important contribution of the Co $d$ states to the valence band top. These states seem to be 
responsible for the specific properties of the Co-based SMSC. The double peak structure of the $d$ states at binding energies from -3.5 to $-2.9 \mathrm{eV}$ due to the enhanced crystal field splitting in the theoretical DOS explains the broad distribution of the $d$ states in the PES spectrum of $(\mathrm{Cd}, \mathrm{Co}) \mathrm{Se}$.

\section{Refezences}

[1] B. Velický, J. Mašek, V. Cháb, M. Surman, K.C. Prince, Festkörperprobleme XXV, 247 (1985).

[2] A. Lewicki, A.I. Schindler, I. Miotkowski, J.K. Furdyna, Phys. Rev. B 41, 4653 (1990).

[3] J. Mašek, Solid State Commun. 78, 351 (1991).

[4] K. Kopalko, B. J. Kowalski, B.A. Orłowski, A. Mycielski, V. Cháb, Acta Phys. Pol. A77, 403 (1990).

[5] T. Moriya, Spin Fluctuations in Itinerant Electron Magnetism, Springer, Berlin 1985.

[6] J. Mašek, this issue..

[7] B.A. Orlowski, B.J. Kowalski, V. Cháb, Phys. Scr. 41, 984 (1990). 